\title{
Lab Counterculture
}

Jessica Hammer

Carnegie Mellon University

Pittsburgh, PA, USA

hammerj@cs.cmu.edu

\section{Alexandra To}

Carnegie Mellon University

Pittsburgh, PA, USA

aato@cs.cmu.edu

Erica Principe Cruz

Carnegie Mellon University

Pittsburgh, PA, USA

ecruz@cs.cmu.edu
Permission to make digital or hard copies of all or part of this work for personal or classroom use is granted without fee provided that copies are not made or distributed for profit or commercial advantage and that copies bear this notice and the full citation on the first page. Copyrights for components of this work owned by others than the author(s) must be honored. Abstracting with credit is permitted. To copy otherwise, or republish, to post on servers or to redistribute to lists, requires prior specific

permission and/or a fee. Request permissions from permissions@acm.org.

CHI '20 Extended Abstracts, April 25-30, 2020, Honolulu, HI, USA.

(C) 2020 Copyright is held by the owner/author(s). Publication rights licensed to ACM.

ACM ISBN 978-1-4503-6819-3/20/04 ...\$15.00.

http://dx.doi.org/10.1145/3334480.3381824

\begin{abstract}
While academic research culture varies across schools, disciplines, and individual labs, the material and mental well-being of both graduate students and faculty are often negatively impacted by systemic factors in academia. Here we unpack these patterns in order to counter the narrative that individualistic solutions can bring about change. We illustrate how focus on quantitative outcomes, perfectionism, competition, time scarcity, power dynamics, bias towards maintaining the status quo, and financial stress contribute to negative lab culture. We describe specific, concrete, and actionable practices we institute in our lab to counter these systemic factors. We end by opening the conversation to other researchers to examine and counter toxic lab culture to promote supportive, inclusive, and ethical research.
\end{abstract}

\section{Author Keywords}

academic research; lab culture; counterculture;

\section{CCS Concepts}

-Social and professional topics $\rightarrow$ Professional topics;

•Human-centered computing $\rightarrow$ Human computer interaction $(\mathrm{HCl})$;

\section{Introduction}

Graduate school places burdens on graduate students and young faculty, framed as expectations for academic 
research success. Graduate students' mental health and well-being suffer [9]; they are overworked [41] and underpaid, struggling financially $[19,39]$. Faculty experience isolation [26] and a desire to leave academia [10]; they work exceptionally long hours [20], doing work not aligned with their research, skills, and desires [11, 23]. Such burdens are compounded for underrepresented minorities $[9,15,30]$ and lead to attrition $[5,31,33,34]$. We believe on average, graduate schools are not currently structured to support the health and well-being of students and faculty.

This paper outlines some systemic graduate school factors that actively harm student and faculty well-being: focus on quantitative outcomes, perfectionism, competition, time scarcity, power dynamics, bias towards maintaining the status quo, and financial stress. We describe some of our lab practices that combat them: celebrating effort over outcomes, elevating the value of community, prioritizing time with each other, establishing protected communication channels, mentoring and facilitating long-term goals, and providing flexible and accessible resources. Our practices are embedded in our context as researchers at an R1 university in a human-computer interaction lab involving games, play, learning, and culture (among other contexts of privilege and marginalization we experience as individuals). We hope that by sharing our policies we can spark a necessary conversation about concrete practices that create inclusive and equitable research labs.

\section{Background}

Graduate students' mental health has been harmed by systemic graduate program characteristics including living conditions, academic engagement, social support, financial confidence, and advising relationships [27]. Students are overworked, feeling pressure to complete research and coursework and solve departmental teaching short- ages [41]; they face six times the rate of depression and anxiety as the general population [9].

Compounding students' financial struggles to meet basic needs (e.g., food security [19]) are reimbursement norms and challenges to maintaining personal relationships and family responsibilities. Students are "expected to spend more money than [they] made in a month on businessrelated expenses over the course of a few weeks" and "expected to be migratory and unattached" [39]. Graduate school conventions exert undue burden, making students stressed, depressed, overworked, underpaid, and unable to prioritize their well-being without compromising their professional success.

Junior faculty, even with power and privilege, bear parallel burdens. They are overworked: $10 \%$ of surveyed faculty report more than 60 hours per week [20]. This is exacerbated by misalignment between how they are expected to spend their time and how they are rewarded for spending it. A 2014 study found faculty spent only $5 \%$ of a typical workweek on primary research and manuscript writing and continued this work during typical leisure hours [11, 24].

They spend a disproportionate amount of time in meetings, answering emails, and on service obligations [11]. Despite long work hours, many researchers feel isolated, anxious, and worried about hitting external quantitative targets [26].

For underrepresented minority students and faculty, these impacts are worse. Female faculty, especially women of color, have higher mentoring, service, teaching, and administrative expectations placed on them than their male peers and also need to produce better work to achieve an equal chance of tenure [30,40]. Graduate student women of color also feel disproportionate pressure to perform, sacrificing their well-being to avoid being cast under stereotypes [15] Female, transgender, and gender-nonconforming graduate 
students experience anxiety and depression at higher rates than males [9].

These struggles often lead to attrition. PhD program attrition surveys showed cumulative completion rates: Engineering was $64 \%$, Mathematics \& Physical Sciences was $55 \%$, and Life Sciences was 63\% [34]. For underrepresented minorities, completion rates were significantly lower: $48 \%$ for Hispanic/Latino students and $40 \%$ for Black/African American students. Nearly $40 \%$ of higher education professionals considered attrition in the past two years [10]. Lack of support, isolation, and racism increase the likelihood that faculty leave academia prematurely $[5,31]$.

\section{Systemic vs. Individualistic Solutions}

From free yoga classes to time-management seminars, the burden of addressing these issues is often placed on the individual. If only students could manage their stress; if only faculty were more willing to say no. We do not buy this story nor think individualistic solutions bring lasting change.

The individualist perspective ignores systemic factors that place graduate students and faculty in untenable positions. For example, power dynamics are a systemic description of relationships between people and institutions. Power dynamics mean a graduate student may compromise their well-being because they cannot refuse an advisor's request. Institutional structures are also systemic. For example, it may be difficult for faculty to manage their time not because any individual request is inappropriate, but because the requesters are unaware of the faculty member's overall workload [16].

Edwards and Roy describe some of these dynamics in their work on perverse incentives in academia [8]. They argue that academia is increasingly interpersonally competitive.

Combined with quantitative measurement techniques, aca- demics are incentivized to perform well by the measures at hand, regardless of whether or not those measures result in good research or happy lives. For example, if researchers are rewarded for their number of publications, and the environment is cutthroat, you end up with an "avalanche" of substandard work [32]. Researchers who game the system will do better, by the system's measures, than those who do not-and in a hypercompetitive environment, researchers who do not game the system may be pushed out entirely.

This shows that apparently maladaptive behavior may be adaptive in the face of a misconstructed system. The individuals involved would not likely choose these outcomes (overwork, mental health issues); they are adaptive responses to the system. Systems may even be deliberately misconstructed to benefit people other than those who must endure them. For example, many factors that hamper graduate students benefit faculty, such as power dynamics that limit graduate students' ability to resist misconduct and reject faculty demands. Creating an academic precariat benefits institutions, who weaken faculty resistance with the possibility of slipping into the underclass [2].

These systemic designs are not accidental, and they cannot be accidentally resisted. The first step is to see them so that we can design against them, which is what we seek to do in the next section.

\section{Dark Patterns}

We draw from multiple sources in identifying key systemic factors-what we might think of as "dark patterns" in the design of academic institutions-that affect the well-being of graduate students and junior faculty $[8,27,36]$. In this section, we argue that these factors generate an environment where student and faculty well-being is compromised. 
Focus on Quantitative Outcomes.

Focus on quantitative outcomes encourages researchers to game the system. While doing this can produce negative externalities like substandard work [32], it can also affect how researchers spend their time: things whose value can be measured, compared to those that cannot, will (sensibly!) take priority.

A paper publication can be measured, put on a CV, and rewarded. But some factors associated with that publication cannot easily be measured. Was the research conducted ethically? Were team members treated with respect? Did students on the project learn something new? If there are no rewards for these types of outcomes, but high rewards for the publication itself, even the most well-intentioned researchers will be tempted to cut corners-and researchers who do will be advantaged over those who do not.

The focus on quantitative outcomes is aggravated by the outcome being typically outside a researcher's control. Paper submissions and grants are reviewed by committees. Teaching evaluations are known to be biased by race and gender [3,22]. This introduces a self-efficacy problem: researchers may feel like they cannot accomplish their goals because harder work will not necessarily help. This has predictably bad effects on well-being.

\section{Perfectionism}

While Edwards and Roy show that hypercompetition can result in shoddy work aimed at gaming the system [8], another maladaptive response is perfectionism. This is due to very long waits for feedback on work from advisors and/or review committees. It makes sense to try to make work bulletproof.

Perfectionism is not just researchers trying to do their best work. That is a healthy academic culture. It is often linked to imposter syndrome and instead creates impossible standards [28]. It also means mistakes are attributed to fixed characteristics of people, rather than seen as part of learning processes or situational factors. When this is the case, it is sensible to avoid failure by any possible means-such as not submitting papers or hiding uncertainty.

\section{Competition}

Hypercompetition means there are not enough resources for everyone to do good work but rather that only the "best" succeed. But both halves of that statement have to be read critically. There may actually be enough resources, but they are being distributed in inequitable ways-leading to perceptions of resource scarcity. And "best" is rarely actually the best but usually biased by external factors such as race, class, gender, etc.

Thoman and colleagues link competition to individualism [36]. Not only is there competition for scarce resources, but the unit of value is the individual rather than a group or team. This leads to a desire for individual credit and can lead to people cutting off access to resources, rewards, and/or credit for those less powerful than they are.

Hyperindividualistic, competitive environments can also make it hard for people to feel included. Why be generous to people when they are threats to your long-term wellbeing? Why trust that someone being kind is actually working to make you included, instead of working for their own agenda? All this can lead to feeling isolated and poor social support, and as a study on well-being in academic environments found, graduate students with less social support are more stressed and depressed [35].

\section{Time Scarcity.}

Funding and publications are not the only resources that can be scarce. Researchers often describe being badly 
pressed for time in a culture where rest is shameful. Because standards for success are so high and resources are scarce, there is a sense of urgency to do more, work more, and succeed more-a known side effect of hypercompetitive environments.

Junior faculty and graduate students work more hours than research shows is productive $[20,41]$. The pervasive sense of hurry urges people to perform busy-ness no matter how busy they are. Adding insult to injury, typically researchers do not feel that their use of time aligns with what they want. For junior faculty, the things they are asked to do as part of their job requirements do not always align with the metrics for success they are measured against. This is, unsurprisingly, worst for women and faculty of color (especially women of color). They are asked to do more work that is not measured nor remunerated and is time-consuming (e.g., mentorship, departmental and community service, teaching, administrative activities) $[37,40]$.

Finally, a sense of urgency and hurry is often antithetical to doing good research, particularly to treating people well while you are doing it [1].

Power Dynamics.

Power dynamics is one area where graduate student and junior faculty experiences diverge, but both are problematic: those with relatively less power do not always know how power works. Graduate students do not get access to faculty decision-making, and junior faculty often do not get introduced to tacit power flows in the department. In both cases, this is not good.

Graduate students are often reliant on a single individual (their advisor) for both their long- and short-term success. This dependence enables professional and sexual predation on the part of faculty who are inclined to such things $[7,18]$. Because faculty can withhold things like recommendation letters or access to data, students may have to comply. Even when this does not rise to the level of actual abuse, the power dynamic between advisors and advisees can be toxic. For example, advisors can easily enable toxic competition in the lab by playing favorites with students who are perceived to be "special."

For junior faculty, the tenure process can be opaque [6, 21]. Many junior faculty worry about voicing controversial opinions or crossing senior faculty because it can lead to their tenure case being sacked. Ultimately this contributes to a culture of power-hoarding where only very few people at the top have full transparency on decision-making and feel empowered to speak-up.

\section{Status Quo Bias.}

Academic institutions benefit from being difficult to enter. At the undergraduate level, prestige is based on low acceptance rates [17]. Doctoral student admission processes are supervised by faculty, and the standard for excellence is opaque and shifts throughout the review process [29]. Faculty cite wanting to admit students who are likely to succeed, but often choose people "like them" who are already insiders in the community [29]. Despite increased rhetoric supporting diversity and inclusion, discourse and action tend to be abstract and high-level without materially engaging with structural inequalities that create those gaps [38]. Even for faculty, the predominant narrative is that hiring is protracted and rigorous (a merit-based system). However, the data shows that faculty hiring typically maintains the status quo: women are placed far less often than men and doctoral prestige tends to be one of the better predictors for hiring [4]. Even when institutes make an effort to hire underrepresented faculty such as women and people of color, they often neglect to provide adequate support for 
them to survive and thrive in an academic culture not built for them [25], leading to poor retention and advancement rates.

Furthermore, when academics struggle, institutions are often unhelpful and unforgiving. In interpersonal cases of abuse, institutions tend to protect themselves first; this slow action protects predators. Students struggling with depression trying to access strained university mental health resources are often told to "take a break". If students leave, they have little to no support resources and no clear path for returning. All of this perpetuates a status quo that often fails to support graduate students and junior faculty.

\section{Financial Stress.}

While there are many factors related to life outside the research context that affect well-being, financial stress is one that bridges both contexts. Financial stress is reported by graduate students as one of the major predictors of wellbeing, and the increasing adjunctification of faculty positions means it affects faculty as well.

In the short-term, students are often asked to bear the expenses of their research career. For example, it is typical to ask students to cover their own conference expenses and wait months for reimbursement (if any) [39]. Students may also have to pay for research expenses, from participant funds to development expenses, if their advisor asks. (We have heard many such stories over the years.) In the long-term, students are often underpaid and cannot afford the high cost of living [19]. Financial stress stems from and affects many aspects of students' lives including but not limited to health insurance costs and overwork due to TAing hours to offset graduate school costs.

For faculty, a competitive funding environment makes it hard to plan ahead. What kinds of support will they get from the institution? If they cannot get funding due to no fault of their own, will their career be seriously compromised? In extension, many faculty worry students they oversee could suffer. This is worse for adjunct faculty who are often under more financial stress: an American Community Survey found that $31 \%$ of adjunct faculty live below or near the poverty line [14].

Finally, both graduate students and junior faculty endure academic precarity. Students worry: will there be jobs? Faculty worry: if they don't make it in this job, will they ever find another? This precarity is a long-term stressor: students and faculty struggle now and worry about the lack of guarantee for a stable future.

\section{Countercultural Patterns}

While these systemic problems affect all people in academia they do not affect everyone equally. As Anatole France puts it, "the law, in its majestic equality, forbids the rich as well as the poor to sleep under bridges, to beg in the streets, and to steal bread" [13]. Marginalized groups are disproportionately affected by these cultural choices, to the point where SURJ calls these patterns of behavior "white supremacy culture" [12].

This happens because of how these patterns interact with pre-existing social systems that are apparently outside of the academic purview. In a world where women carry the bulk of unpaid work, time scarcity will affect them more than it affects men. In a world where Black and other racial minority families have far less generational wealth than White families, financial stress disproportionately affects Black students. In a world where neurodivergence is punished, perfectionism puts more pressure on neurodivergent than on neurotypical students to conform. 
In other words, even awful systems are not neutrally awfuland leaving them in place continues to oppress the most disadvantaged. Therefore you need active countercultural patterns that both take into account these dark patterns of academic life and recognize the realities of who is most affected by them and why. These patterns can be dealt with at different institutional levels, but systemic problems need systemic solutions-whether that is within a lab, a department, or the entire field.

Here we describe specific practices we use in our lab to counter some of the dark patterns we have observed and experienced in academia. We look at graduate students and junior faculty together because the well-being of one is often treated as something that comes at the expense of the other. We think that with solidarity as a key goal of countercultural pattern-building, we can create systems that help everyone. These are, of course, not the only solutions, and we hope that these examples open a necessary conversation and invite others to share their practices.

\section{Celebrating Effort over Outcomes}

We counter focus on quantitative outcomes and perfectionism by celebrating lab members' quality work as iterations towards excellence rather than the outcomes of those efforts. For example, we celebrate submissions rather than acceptances (for papers, grants, job applications, etc.). Submissions are not a scarce resource. In practice, this is asking graduate students to share when they have submitted a paper in advising and/or lab meetings. When a paper is submitted, everyone present claps and cheers. Additionally, it means changing language around paper acceptances. Instead of describing them as evidence that the work is good, we say that we are glad that other people have recognized the value of the work. This reminds students that work can be good even if it is rejected, allows re- flection on the paper writing process, and enables focus on doing more excellent work instead of on outcomes-based reputations.

Also, as many people in the lab can submit a paper as desired; someone's submission does not detract from anyone else's opportunity to submit. Additionally, submissions are within graduate students' control. This allows each student to develop a sense of self-efficacy; they know working harder or smarter can change whether they are able to submit, even if the ultimate fate of the paper is in the hands of reviewers.

Celebrating effort over outcomes makes it clear that perfection is not expected, which is important early in graduate students' careers. Mistakes are not attributed to any lab member's lack of ability; instead, they are treated as material for iteration and as an expected step in the learning process. For example, students are congratulated for completing research tasks before addressing errors and omissions, often with language like "now let's improve this work". Treating imperfect work as a step toward amazing work enables lab members to share their progress without fear, enabling constructive feedback at all stages of the working process and a healthier lab culture experience.

What makes this work? Our lab cannot afford to not publish. Not caring about publications is a luxury that injures the most vulnerable members of the lab the most. But focusing on acceptances is stressful and can emphasize competition in the lab-especially when the number of acceptances is out of our control and low acceptance rates are often treated as signals of venue quality. Consistent practice of genuine celebration of work done and learning from mistakes via applause, kind words, and helpful feedback drive our healthy lab culture. Faculty have to adopt this too; if they only celebrate publications, then students 
will not be able to put aside the standards of the larger community. Instead, they may experience the focus on submissions as condescending or as a signal that faculty think they cannot handle the "higher" standard.

\section{Elevating the Value of Community}

We counter competition by asserting that supporting our lab and community members is the most important way we spend our collective and individual time. Our core practice in weekly lab meetings is to ask, "How can the lab help you?" This question is integrated into many activities, but whoever is facilitating saves time to ask each member present to answer it. While every member may ultimately be engaged in their own research, resources such as funding, equipment, and even time are distributed equitably and to whomever needs them when they need them (as opposed to say, focusing on the lab member with the highest profile work). A lab member's social and professional "status" is elevated not through publications but rather pro-social actions such as helping other lab members or doing service for the lab.

What makes this work? Our lab's on-boarding process informs incoming members of how the community functions, how resources can be accessed and how they are allocated, and how individual standing is built upon effort and service for the lab rather than external metrics such as publication quotas. Lab members know they do not have to compete for resources that they rightfully have equitable access to, and this strengthens the community and provides a healthier research lab culture.

\section{Prioritizing Time with Each Other}

We counter time scarcity by prioritizing and scheduling time together during the workweek. This puts institutional power (e.g. the faculty member saying "do this," having it appear on people's calendars) in service of our collective goals. It also gives people a social excuse. No matter how pressed they feel for time, they can always say "well this is what our lab does" or "this is what my advisor wants me to do" and have evidence for it. Lab meetings, as described earlier, use this technique to prioritize helping each other with research; optional lab bonding time uses the same technique to help us see each other as whole human beings who have needs outside of research success.

Biweekly, optional lab bonding time allows us to make a statement with our time and our calendars during the workweek to say, "I see you as a whole person, not just as a research machine, and I care about you as that whole person". During this time, junior members of the lab take only the first 10 minutes to vent about work stress and then spend the rest of the time getting to know each other, eating snacks, playing games together, and taking a complete hiatus from work. We prioritize rest, developing community relationships that are not instrumentalized, and joy. Opting out of this does not put students at a disadvantage; work is not involved and the lab respects students' time and how they choose to spend it. Faculty support this by surfacing and communicating this practice to all new lab members, dedicating everyone's time publicly, and by providing a budget.

What makes this work? Putting more meetings on someone's calendar is counterproductive. What makes this work is that faculty actually schedule the week's obligations around it, not just add it to students' schedules and expect them to (individually) find a way.

Establishing Protected Communication Channels We counter toxic and inequitable power dynamics by establishing protected communication channels for feedback. Providing feedback to supervisors can come with risks of backlash, so faculty solicit anonymous feedback at least 
once per semester. The form has three questions: 1) What is something l'm doing well as your supervisor? 2) What is one thing I could improve, and how? And 3) Is there anything else you think I should know? While respondents are invited to identify themselves if it would lead to a necessary deeper conversation, the default is that feedback is anonymous.

What makes this work? This feedback style comes from a culture of iterative improvement (e.g. from $\mathrm{HCl}$ and games). The framing shifts us away from viewing feedback as criticism and towards seeing feedback in all forms as useful. If something is working for you, great, we will keep doing that If something is not working for you, it is imperative to let us know so we can change to best serve our work dynamic. The anonymous nature of the feedback alleviates concern over both conscious and unconscious backlash

\section{Mentoring and Facilitating Long-Term Goals}

We counter bias towards maintaining the status quo of academia by mentoring people in the lab beyond the immediate goals of our research. In publicizing opportunities in the lab, we emphasize hiring people who are excited and willing to learn new skills and perform interesting research rather than expecting pre-existing expertise. This helps us recruit more diverse candidates. We also encourage graduate students to supervise teams of undergraduate researchers to give opportunities to many students. Finally, every supervisor in the lab (graduate student or faculty) is trained to discuss long-term goals with mentees early on. We always set at least one goal for the semester to align with a students' professional advancement (e.g., create a portfolio piece for an industry position, co-author a research publication, develop a new technical skill).

What makes this work? By hiring more inclusively for the lab, we widen the pipeline of students entering both our lab and our field more broadly. We use a mentorship model that focuses on the specific needs and goals of the students we work with rather than treating them as replaceable cogs in a machine. This professionally uplifts all students we supervise.

Providing Flexible and Accessible Resources We counter financial stress for students by putting structures in place so students should never personally take on work-related expenses. We have a lab credit card that is designated for use for academic conference travel expenses (e.g., flights, lodging, registration), equipment, software, and materials for studies. We also maintain a flexible lab-wide fund for minor expenses like compensation for human-subjects research participants. We strive to eliminate the need for students to use their own money to pay for work-related expenses and then wait for weeks if not months to be reimbursed.

What makes this work? By providing structured financial support for research and lab expenses, students are never required to take on financial burdens in order to be successful researchers. This practice visibly acknowledges the financial burden of academia and ensures we do not apply differential pressure to people from different socioeconomic backgrounds.

\section{Conclusion}

In this paper we have identified some of the major dark patterns at play in academia and provided a few countercultural patterns-specific policies our lab uses-that work against them. We recognize that the examples we have provided may seem small, and we know that there has to be change beyond our own lab in order to disrupt the patterns we identify. However, we nonetheless believe there's value in "digging where you stand." First, we've found that 
it takes practice and careful thought to disrupt the dark patterns, even on a small scale. Practicing in the lab (which is contingent on advisor willingness to enable countercultural patterns but can be initiated by students and ultimately requires enactment by all lab members) helps us all be prepared to continue that disruption when we have more power. Second, the experiences that both students and junior faculty have in our lab help shift the window of what's considered normal and acceptable. We have found that our peers, seeing what is possible, become more able to see dark patterns and also more willing to resist them. While each individual change is relatively small, the sum of our countercultural choices (including many that we could not describe in this paper for lack of room) creates a highly atypical experience overall. That divergence is itself of value. Finally, we believe it is important to live our own values starting now. While the nature of academic institutions and society as a whole may need to change, that doesn't exempt us from our responsibilities to others, particularly those who have less power in a given situation than we do.

What has it felt like for us to do these things? Each of us comes from a different position within the lab. As a first year graduate student, Cruz feels our lab culture has "defanged" her previous impression of academic research culture. She has more capacity to learn effectively and does better work because she does not worry about impossible standards for the quality of her first tries, expectations about working until she passes out, and not being seen as a whole person. As a graduate student who is ABD (all-but-dissertation), To feels prepared to adapt our lab policies to a new department as future faculty. As her power has shifted over the course of the PhD program, she has seen how using transparent policies that are justice-oriented has reduced her likelihood of making biased and exploitative decisions towards students she supervises while giving her freedom and structure to work on projects she is passionate about. As faculty, Hammer feels the whole lab is enormously more productive; students do better work when they are not worried about paying rent or afraid that she is going to judge them for putting a step wrong. It is also a joyful place for all lab members to be. It is a pleasure for her to go to work every day with people who take pleasure in being there.

On a pragmatic level, we have found that these practices have improved our well-being and quality of life, without compromising our academic excellence. For example, lab members typically work a 40 hour week, and take real noemail no-work vacations. When a lab member needs help, the community rallies around them with everything from help with academic tasks to delivering meals. At the same time, we sustain a high rate of publication in top venues (e.g. eight papers from the lab at this year's $\mathrm{CHI}$ alone) and regularly win awards for our creative work. Our success illustrates that academia does not have to be a zero-sum game. Junior faculty do not have to make their careers on the backs of exploited graduate students, nor do they have to martyr themselves to be just. Instead, we can design systems that help us all succeed.

We recognize we are not the only ones resisting these dark patterns. We have spoken to other researchers who actively design their labs for well-being and inclusion. We chose to write this paper to begin a conversation, so that we can begin to disseminate best practices in this area and evaluate what is most effective. We very much look forward to discussing with others what we can do now in order to shift the system in the long run. We can all seek justice together to create long-term sustainable research cultures that include everybody, not just the people least disadvantaged by the dark patterns of academia. 


\section{REFERENCES}

[1] C Daniel Batson, Pamela J Cochran, Marshall F Biederman, James L Blosser, Maurice J Ryan, and Bruce Vogt. 1978. Failure to help when in a hurry: Callousness or conflict? Personality and Social Psychology Bulletin 4, 1 (1978), 97-101.

[2] Marc Bosquet. 2008. How the University Works. Higher Education and the Low-Wage Nation (2008).

[3] Kerry Chávez and Kristina MW Mitchell. 2019. Exploring Bias in Student Evaluations: Gender, Race, and Ethnicity. PS: Political Science \& Politics (2019), $1-5$.

[4] Aaron Clauset, Samuel Arbesman, and Daniel B Larremore. 2015. Systematic inequality and hierarchy in faculty hiring networks. Science advances 1,1 (2015), e1400005.

[5] Karen L Cropsey, Saba W Masho, Rita Shiang, Veronica Sikka, Susan G Kornstein, Carol L Hampton, Committee on the Status of Women, and Medical College of Virginia Campus Minorities, Virginia Commonwealth University School of Medicine. 2008. Why do faculty leave? Reasons for attrition of women and minority faculty from a medical school: four-year results. Journal of Women's Health 17, 7 (2008), 1111-1118.

[6] Jennifer Diascro. 2019. Failure in the Tenure Process: We Can Do Better. PS: Political Science \& Politics 52 , 1 (2019), 48-51.

[7] Lillian T Eby. 2007. Understanding relational problems in mentoring. The handbook of mentoring at work: Theory, research, and practice (2007), 323-344.

[8] Marc A Edwards and Siddhartha Roy. 2017. Academic research in the 21st century: Maintaining scientific integrity in a climate of perverse incentives and hypercompetition. Environmental Engineering Science 34, 1 (2017), 51-61.

[9] Teresa M Evans, Lindsay Bira, Jazmin Beltran Gastelum, L Todd Weiss, and Nathan L Vanderford. 2018. Evidence for a mental health crisis in graduate education. Nature Biotechnology 36, 3 (2018), 282-284.

[10] Anna Fazackerley. 2019. 'It's cut-throat': half of UK academics stressed and $40 \%$ thinking of leaving: Frequent rejection and a loss of control are making university staff isolated and ill, new research shows. https:

//www . theguardian.com/education/2019/may/21/ cut-throat-half-academics-stressed-thinking-leaving. (May 2019).

[11] Colleen Flaherty. 2014. So Much to Do, So Little Time: Research shows professors work long hours and spend much of day in meetings and on administrative tasks, and much of their time alone.

https://tinyurl.com/pfcck67. (Apr 2014).

[12] Showing Up for Racial Justice (SURJ). 2019. White Supremacy Culture. (Dec 2019).

https://www. showingupforracialjustice.org/ white-supremacy-culture.html

[13] Anatole France. 1910. The red lily. Vol. 1. J. Lane.

[14] Caroline Frederickson. 2015. There Is No Excuse for How Universities Treat Adjuncts: Students are paying higher tuition than ever. Why can't more of that revenue go to the people teaching them? https: //www . theatlantic.com/business/archive/2015/09/ higher-education-college-adjunct-professor-salary/ 404461/. (2015). 
[15] Kenneth D Gibbs Jr and Kimberly A Griffin. 2013. What do I want to be with my PhD? The roles of personal values and structural dynamics in shaping the career interests of recent biomedical science PhD graduates. CBE-Life Sciences Education 12, 4 (2013), 711-723.

[16] Philip Guo. 2014. Why academics feel overworked: (my totally unscientific folk theory).

http://www.pgbovine.net/

why-academics-feel-overworked .htmg. (Nov 2014).

[17] Anemona Hartocollis and Kate Taylor. 2019. Elite Colleges Announce Record Low Admission Rates in Wake of College Cheating Scandal.

https://www.nytimes. com/2019/03/29/us/ college-admissions-rates.html, (Mar 2019).

[18] W Brad Johnson and Jennifer M Huwe. 2002. Toward a typology of mentorship dysfunction in graduate school. Psychotherapy: Theory, Research, Practice, Training 39, 1 (2002), 44.

[19] Karen Kelsky. 2019. Boston U Dean to Struggling Grad Students: Go To the Food Pantry - Guest Post. (Dec 2019). https: //tinyurl. com/vghjgzf

[20] Henry Kronk. 2018. Professors Are Overworked and Poorly Paid by a Troubled System of Higher Education, Top Hat Survey Finds. (Aug 2018). https://tinyurl.com/unt5v31

[21] Janet H Lawrence, Sergio Celis, and Molly Ott. 2014. Is the tenure process fair? What faculty think. The Journal of Higher Education 85, 2 (2014), 155-192.

[22] Lillian MacNell, Adam Driscoll, and Andrea N Hunt. 2015. What's in a name: Exposing gender bias in student ratings of teaching. Innovative Higher Education 40, 4 (2015), 291-303.

[23] David Matthews. 2018. If you love research, academia may not be for you. (Nov 2018).

https://www.timeshighereducation. com/blog/

if-you-love-research-academia-may-not-be-you

[24] Kelly E Matthews, Carmen Garratt, and Doune Macdonald. 2018. The higher education landscape: trends and implications. (2018).

[25] Susana M Muñoz, Vincent Basile, Jessica Gonzalez, Daniel Birmingham, Antonette Aragon, Louise Jennings, and Gene Gloeckner. 2017. (Counter) narratives and Complexities: Critical Perspectives From a University Cluster Hire Focused on Diversity, Equity, and Inclusion. Journal of Critical Thought and Praxis 6, 2 (2017), 1.

[26] Tim O'Brien and Dennis Guiney. 2018. Staff Wellbeing in Higher Education: A research study for Education Support Partnership. London: Education Support Partnership (Dec 2018)

[27] Galen Panger and T Janell. 2014. Graduate student happiness \& well-being report. The Graduate Assembly, University of California Berkeley (2014).

[28] Anna Parkman. 2016. The imposter phenomenon in higher education: Incidence and impact. Journal of Higher Education Theory and Practice 16, 1 (2016), 51.

[29] Julie R Posselt. 2014. Toward inclusive excellence in graduate education: Constructing merit and diversity in $\mathrm{PhD}$ admissions. American Journal of Education 120 , 4 (2014), 481-514. 
[30] José Rodríguez, Kendall Campbell, John Fogarty, and Roxann Williams. 2014. Underrepresented minority faculty in academic medicine: A systematic review of URM Faculty Development. Family medicine 46, 2 (2014), 100-104.

[31] José E Rodríguez and Kendall M Campbell. 2013. Ways to guarantee minority faculty will quit academic medicine. Acad Med 88, 11 (2013), 1591.

[32] Paul E Smaldino and Richard McElreath. 2016. The natural selection of bad science. Royal Society open science 3, 9 (2016), 160384

[33] Robert Sowell, Jeff Allum, and Hironao Okahana. 2015. Doctoral initiative on minority attrition and completion. Council of Graduate Schools.

[34] Robert S Sowell, Nathan E Bell, Sheila Nataraj Kirby, and Scott Naftel. 2009. Ph.D. Completion and Attrition: Findings from Exit Surveys of Ph.D. Completers. Council of Graduate Schools.

[35] Tracy Stecker. 2004. Well-being in an academic environment. Medical Education 38, 5 (2004), 465-478.

[36] Dustin B Thoman, Elizabeth R Brown, Andrew Z Mason, Allen G Harmsen, and Jessi L Smith. 2015. The role of altruistic values in motivating underrepresented minority students for biomedicine. BioScience 65, 2 (2015), 183-188.

[37] Caroline Sotello Viernes Turner. 2002. Women of color in academe: Living with multiple marginality. The Journal of Higher Education 73, 1 (2002), 74-93.

[38] Richard Waller, Nicola Ingram, and Michael RM Ward. 2017. Higher education and social inequalities: University admissions, experiences, and outcomes. Routledge.

[39] Linda Wang and Celia Henry Arnaud. 2018. Grad school, in students' own words: Chemistry grad students share their diverse experiences. https: //cen.acs.org/education/graduate-education/ Grad-school-in-students-own-words/96/i36. (Sep 2018). Issue 36.

[40] Kate Weisshaar. 2015. Publish and Perish?: An Assessment of Gender Bias in Academia. Unpublished manuscript (2015).

[41] William Douglas Woody. 2004. Universities, psychology departments, and the treatment of graduate students. In Essays from E-xcellence in Teaching 2003, William Buskist, Vincent W Hevern, Bryan K Saville, and Tracy Zinn (Eds.). Vol. 3. Society for the Teaching of Psychology, Chapter 3, 28-32. 


\section{Commentary}

For alt.chi paper

Lab Counterculture

\section{Max Willis}

ITI / LARSyS and Madeira

Interactive Technologies Institute

Funchal, Portugal

max.willis@m-iti.org
This is an interesting and thoughtful paper, if indeed at first a depressing read. The phrasing of the challenges (no, the problems) as dark patterns is unique, and neatly encapsulates the idea that these are systemic issues that cannot be adequately solved through individualistic solutions. The dark patterns are clear, and the paper elaborates each in short form while still managing an excruciating detail in its overview. I am sure this will serve many as an excellent starting point to delve deeper into the various issues, and indeed seems like an abbreviation of more extensive research done by the authors. The second part of the document, in which the authors' introduce their own lab practices, is more uplifting. These new behaviors do indeed sound progressive and impactful, what could lead to a much more comfortable working environment.

I cannot fault the writing and the paper is sure to drive some interesting discussion, to which I would add some thoughts on the 'everyone is special' attitude in applauding submitted papers, which is nice but may not actually foster excellence, even as asking in follow-up 'now how do we make it better?' seems to be, asking for excellence, if not perfection. Also the 'work together, play together' concept seems forced, pressuring people to blend work and social in ways that not everyone is comfortable doing, thus potentially penalizing people who are not particularly social by default. The reporting system, asking for feedback is not innovative or unusual, and there is no reflection whether or not people in the lab actually believe the reporting system is useful, effective, and indeed anonymous.

With such practices being introduced I have no doubt your lab culture is flourishing. It would be interesting however, if you could share some data or insights on your lab's academic, publishing and research productivity. Such cold and inhuman metrics are clearly what you are trying to counter, but for other institutes and labs who might be interested in the changes you suggest, some evidence that happy practices will not collapse their research output could be encouraging.

For me the beginning of the section 'countercultural patterns' references white supremacy, which I think is misleading. I do not doubt that in many cases this link can be in effect, however I feel the discussion would benefit by elaborating rather in terms of intersectionality, as the following paragraphs seem to do without actually borrowing the word. It leaves me question, why the explicit terminology of racism rather than the one from gender studies, which could be more widely encompassing.

I think this is a overall solid contribution, it touches on very important issues that are not adequately addressed in academic communities, and the authors describe some real world solutions from their own practices. I appreciate the brief personal anecdotes at the end, that share three different attitudes towards the lab's progress. 